\title{
Improve Google Loon's Indoor LTE Coverage in Rural Africa by Using Passive Repeaters
}

\author{
P. Lynggaard \\ CMI, Aalborg University Copenhagen, Copenhagen, Denmark \\ E-mail:perlyn@es.aau.dk
}

Received 4 August 2017;

Accepted 14 August 2017

\begin{abstract}
Providing LTE connectivity to rural areas in Africa is a very challenging task that has been addressed with many different solution-models over the years. One model that seems to offer a sustainable business case is based on the Google 'Loon' LTE project, which provides many advantages. This solution, however, has a major disadvantage in form of a limited coverage, especially within the homes where the wall attenuation disrupts the signal. This paper provides a solution to this problem in form of deploying a cheap, simple, and passive repeater that enables indoor communication. By simulating the LTE radio-link between a Google 'Loon' base station and a user positioned inside a home, it has been found that the passive repeater lowers the wall attenuation considerably and thereby enables indoor communication.
\end{abstract}

Keywords: LTE-coverage, Passive-repeaters, Google-Loon, wall-attenuation and path-loss.

\section{Introduction}

Today almost half of the population in Africa (500 millions) has subscribed to mobile services. This number is expected to increase to 750 million subscribers by 2020 [1] where a large proportion of these are mitigating to

Journal of NBICT, Vol. 1, 91-106.

doi: 10.13052/nbjict1902-097X.2017.006

(c) 2017 River Publishers. All rights reserved. 
mobile broadband services. Especially factors such as network roll out, low cost phones, and new strategies from the mobile operators are driving this tendency [1]. The number of smart phones that support mobile broadband services in Africa has passed 200 millions which strongly reflects its uptake in the established mobile market. However, by 2020 a significant proportion of the population in Africa will still be unconnected. In particular, the low coverage of $4 \mathrm{G}$ technologies is a challenge. Some initiatives have been deployed to increase the adoption-rate of $4 \mathrm{G}$ technologies such as smart phones for less than $50 \$$ and cheap solar-as-a-service business models [1].

To overcome the challenge of the low $4 \mathrm{G}$ coverage, Google has launched a project that utilizes balloons equipped with eNodeB base-stations to provide LTE access in rural areas. This project has launched balloons to the stratosphere since 2013 with the purpose of creating a self powered balloon based network of LTE eNodeB base-stations that provide coverage to rural areas. It is possible to connect to these balloons from a simple LTE mobile phone located on ground.

One of the key challenges in the Google 'Loon' project is the long range (approximately $20 \mathrm{~km}$ ) between a LTE mobile phone and the nearest eNodeB base-station located at a balloon. The downlink path can be established by increasing the eNodeB transmit-power for the extra costs of increased solar panel area, increased system price, and increased system complexity. However, the uplink from the LTE mobile phone to the eNodeB base-station is a significant challenge due to the relatively low transmit-power provided by LTE mobile phones. The easiest way to handle this is to use high-gain antennas on the eNodeB base-station. But high-gain antennas come for the cost of reduced opening angle, i.e. their beam-width is limited. This means that either the balloons cover a small area with high signal strength or they cover a larger area with a low signal-strength. It seems as Google has chosen to cover the large area for the costs of low field strength, which means that connectivity from inside the homes requires an external antenna mounted at the roof $[2,3]$.

This paper focuses on solving this challenge by using passive repeaters to amplify the indoor signal. A passive repeater consists of two antennas one outside and one inside which is connected by a cable and a small passive electronic box (a phase-shifter). The outside antenna can be a simple high gain antenna (e.g. a Yagi-antenna) whereas the indoor antenna can be a microstrip-array mounted on the wall where it can be hidden, e.g. behind a curtain. It is noted that the estimated price level for these components is less than a few hundred dollars. 
The methodology used in this research was to derive the formulas for calculating the required signal strength in order to connect a LTE mobile phone with a Google 'Loon' eNodeB base-station. These formulas include distance, wall attenuation, and passive repeater equations. They have been used to construct a simulation model which runs in a mathematical tool. The outcomes of the model are elaborated, presented, and discussed in this paper.

It has been found that indoor coverage is a problem which is caused by the low field strength used by the eNodeB balloons and that the house walls attenuate the signal too much to establishing a connection with a high datarate. However, it turns out that the deployment of a simple passive repeater outweighs the wall losses and thereby increases the indoor coverage, i.e. it enables LTE services from indoor environments.

\section{Related Works}

Many different solutions have been analyzed to deal with the challenges of making LTE communication possible in rural areas. One of these is the GSMA group [1] which addresses the development of the rural areas and how to increase the digital inclusion by using economic benefits. They predict this will improve broadband infrastructure and services in the long run. Another work by Lynggaard [4] focuses on providing internet to the rural areas in Africa by using passive repeaters which connect to WiFi towers placed in the center of the villages. This paper is based on a solution offered by BlueTown [5] which deploys micro-operators in the rural villages that connects WiFi base-stations wirelessly to the existing Internet backhaul. The end-user then connects to the access-point offered by these base-stations by using WiFi enabled devices like, e.g. mobile phones (smart phones). Similarly, Simba et al. [6] presented a survey where they discuss the usage and deployment of WIMAX and 3G technologies in Africa. They did not present any specific solution, but elaborates advantages and disadvantages in different approaches.

A white paper by Giliant et al. [7] suggests an approach to provide LTE access to rural areas in Africa by deploying distributed eNodeB basestations and connect these to the backhaul by satellites. Their suggestion is based on a shared revenue model. A work performed by Mardeni et al. [8] discusses a concept based on Wireless Regional Access Networks (WRAN). They compare a WRAN based solution in Zimbabwe with other 
technologies such as WiMAX and mobile cellular with respect to bandwidth and the number of subscribers. They found that WRAN solutions provide more bandwidth and lower prices than WiMAX solutions. However, this solution has a challenge with interferences from the existing TV stations that utilize the spectrums.

\section{The Google ‘Loon’ Project}

The Google 'Loon' project is a development project with the mission of providing LTE access to rural and remote areas. Today, the project is handled by the semi-secret company X (formerly Google X) which restricts the public available information. However, the project has a public website [9] and is covered by other media such as CNBC [10]. From these sources it seems as the project is very mature with an auto-launcher in Puerto Rico that is able to launch a balloon ever 15 minutes, a flight record of more than $122000 \mathrm{~km}$, and a newly tested navigation system based on artificial intelligence.

From a technical perspective the Google 'Loon' project covers a network of connected balloons which provide LTE connectivity to rural areas worldwide. This network consists of a ground station with internet access which connects to a transponder located on the nearest balloon (Figure 1).

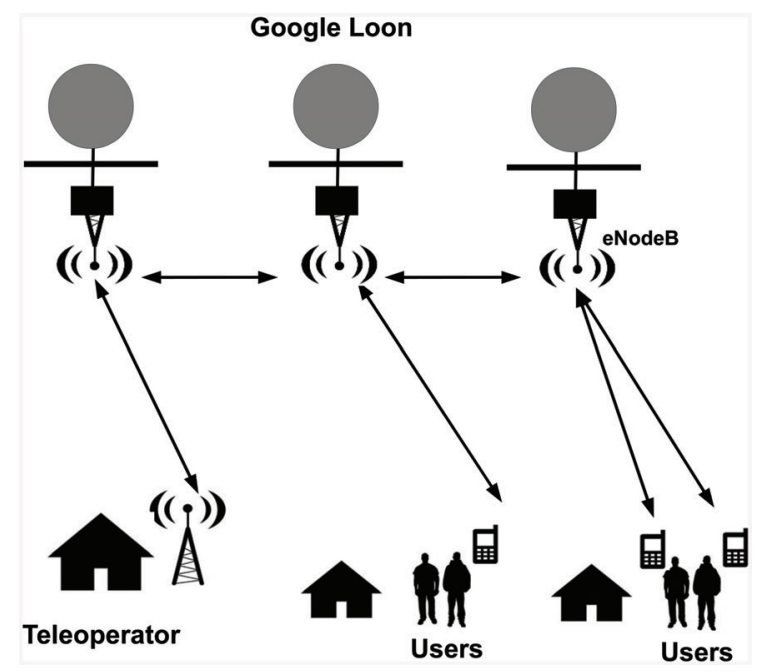

Figure 1 Key elements in the Google 'Loon' project. Users are connected to a teleoperator through a chain of interconnected balloons where each of these carries a LTE-base station (i.e. an eNodeB). 
This balloon is part of a transponder-chain that interconnects all the balloons, i.e. a wireless channel is created between the balloons, which is used to transport LTE communication. In addition, each transponder is equipped with a ground-antenna which provides base station (eNodeB) connectivity for the LTE phones.

These balloons are located approximately 20 kilometer over ground in the stratosphere where they drift with the wind. To keep the balloons at a fixed spot they are controlled by changing their heights and thereby position them in wind streams which have the desired directions.

\section{Passive Repeaters}

A passive repeater consists of two antennas that are connected by a cable and a phase-shift device which passes through the wall of a house. When a signal is received at the outside antenna it is carried through the wall by the cable and the phase-shift device. This signal is then superimposed to the signal that penetrates the wall by using constructive interference and finally this combined signal is retransmitted by the antenna inside the home (Figure 2).

In general, a passive repeater provides some advantages such as higher indoor power level, no power consumption, and it offers a simple construction; however, the cost of the components is an important factor which must be taken into consideration. The impact of this factor can be reduced

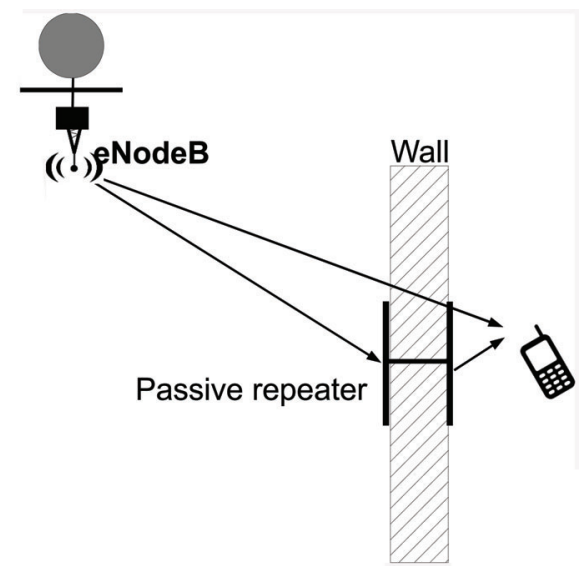

Figure 2 A passive repeater connects the ray transferred from outside to inside with the ray passing through the wall and thereby change the indoor signal level. 
by using integrated antennas which can be mass produced for a limited cost. Similarly, the uniformity of the signal coverage is a challenge because the two superimposed signals will change phase and amplitude as a function of the distance from the indoor emitting antenna. These changes cause "repeating spots" in the home where the signals align in either a constructive or in a destructive way.

\section{The Simulation Model}

This section models, simulates, and elaborates the concept of combining the Google 'Loon' balloons with a passive repeater to increase the indoor coverage and the LTE data-rate.

\subsection{Path Loss}

As discussed the Google 'Loon' project uses balloons with an onboard eNodeB base-station which is placed approximately $20 \mathrm{~km}$ over ground. This distance and the beam width of the antennas used give some challenges. The large distance imposes a huge path-loss for both the uplink and the downlink paths. The downlink path from the eNodeB to a LTE mobile phone can be overcome by using sufficient transmit-power; however, sufficient transmit-power means more power drained from the power-source of the balloons. This power-source is based on solar-cells and rechargeable batteries which provide limitations in form of weights, sizes, and performance. Similarly, the uplink from a LTE mobile phone to the eNobeB is challenging in form of the limited transmitpower available from a LTE smart phone (approx. $23 \mathrm{dBm}$ ) [11]. By using this transmit-power in the commonly well known Friis transmission formula (1), $[12,13]$ the path-loss challenge can be calculated.

$$
\frac{P_{r}}{P_{t}}=G_{r} G_{t}\left(\frac{\lambda}{4 \pi R}\right)^{2}
$$

Where

- $P_{r}$ is received power with antenna gain $G_{r}$

- $P_{t}$ is transmitted power with antenna gain $G_{t}$

- $\lambda$ is wavelength of the signal

- $R$ is the distance between receiver and transmitter.

Assuming the LTE signal is $2.6 \mathrm{GHz}$ (used in Africa); the range between the LTE mobile phone and the eNodeB is $20 \mathrm{~km}$; the transmit power used by the LTE mobile phone is $23 \mathrm{dBm}$; the antenna integrated into the LTE 
mobile phone gives $3 \mathrm{~dB}$ 's gain; and the eNodeB antenna has a gain of $12 \mathrm{db}$ the received power at the eNodeB antenna can be calculated. It is noted that the eNodeB antenna gain is approximated because to the author's knowledge there is no public information available. The only information provided is that the diameter of the circular area on ground is $40 \mathrm{~km}$. By assuming that the limit of this area is the opening-angle of the antenna $(-3 \mathrm{db}$ points), the field strength is equally distributed, and ignoring the curvature of the earth the gain can be approximated as (2) [14]:

$$
G=\frac{16}{\sin ^{2}\left(\tan \left(\frac{\xi_{1}}{\xi_{2}}\right)^{-1}\right)}
$$

Where $\zeta 1$ is the circular radius on ground $(20 \mathrm{~km})$ and $\zeta 2$ is the distance between the receiver and the transmitter $(20 \mathrm{~km})$.

By using Equation (1) and Equation (2) a plot of the received power at the antenna of the eNodeB located at the balloon from a typical LTE mobile phone can be calculated (Figure 3).

As shown in Figure 3 the received power levels at the antenna of the eNodeB located at the balloon from a typical LTE mobile phone is in the range of approx. $-89 \mathrm{dBm}$ to $-91 \mathrm{dBm}$ within a distance range of 20 to $30 \mathrm{~km}$. These levels are close to the sensitivity limit (dashed line in Figure 3),

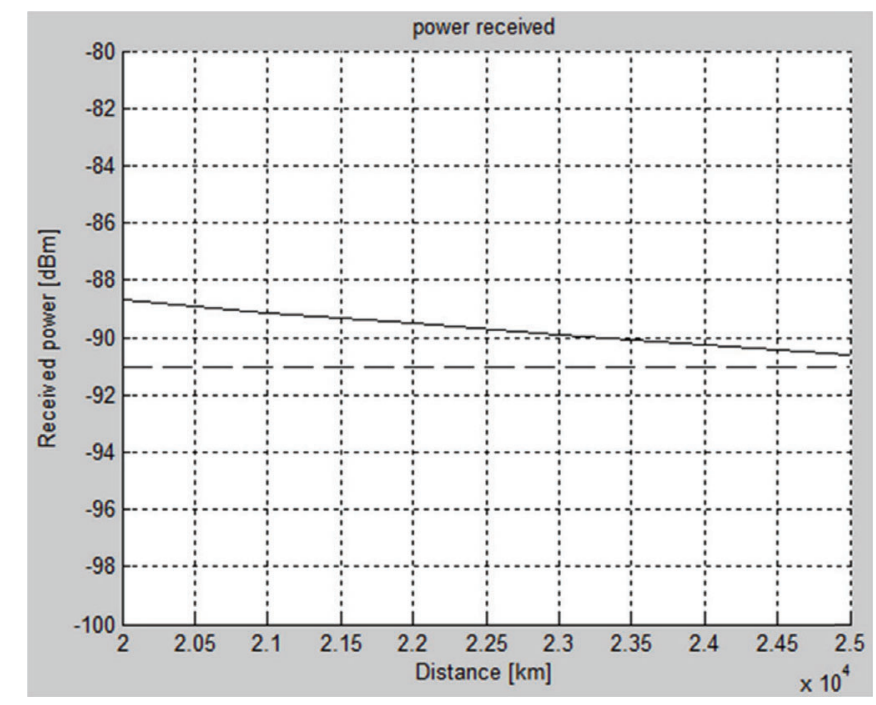

Figure 3 Received power at the antenna of the eNodeB located at the balloon from a typical LTE mobile phone (the solid curve) according to Friis formula (1). The dashed line indicates the $-91 \mathrm{dBm}$ level. 
which is at approx. $-91 \mathrm{dBm}$ if the following parameters are used: QPSK, $20 \mathrm{MHz}$ bandwidth, FDD in the different E-UTRA bands [11]. This means that even small losses in the channel from factors such as interference, weather factors, depolarization losses, and antenna pointing losses can bring the signal to a critical level. In addition, it cannot be assumed that the balloon will stay exactly at the intended position, because their position regulation mechanism is subjected to some uncertainty. Hence, if the balloon moves e.g. $10 \mathrm{~km}$ in one direction the path length ( $\mathrm{R}$ in Friis formula (1)) will increase to approximately $22 \mathrm{~km}$ which decreases the received level at the eNodeB (Figure 3).

As discussed, the communication channel from the LTE mobile phone to the eNodeB (uplink) located at the Google 'Loon' balloon is at the limit for running high data-rates communication. Fundamentally, this means that it will not be possible to connect to the eNodeB from indoor because the walls impose additional attenuation often in the range from 6 to $10 \mathrm{~dB}[4,15]$.

\subsection{Wall Loss}

Focusing on the losses in the path from the LTE mobil phone to the eNodeB located on a balloon the path-loss is the most significant factor; however, the wall penetration loss is contributing considerably as well. This loss can be modeled by the complex electrical parameters of the building materials. In developing countries like Africa common building materials are wood, mud-bricks, rammed earth, and stones [4].

A practical way to model these walls is assuming they are homogeneous plates with uniform thickness and uniformly distributed parameters. Given these assumptions the transmission and reflection coefficients can be described (3) $[4,16]$.

$$
T_{E}=\frac{\left(1-R^{2}\right) P_{d} P_{b}}{1-R^{2} P_{d}^{2} P_{a}} \quad R=\frac{\cos \theta_{i}-\sqrt{\varepsilon_{r}-\sin ^{2} \theta_{i}}}{\cos \theta_{i}+\sqrt{\varepsilon_{r}-\sin ^{2} \theta_{i}}}
$$

Where

- $P_{d}$ is $\exp \left(-\mathrm{jk} \mathrm{k}^{\prime}\right)$

- $P_{b}$ is $\exp (\mathrm{jkb})$

- $P_{a}$ is $\exp \left(\mathrm{jk} 2 \mathrm{l} \sin \theta_{t} \sin \theta_{i}\right)$

- $\mathrm{k}, \mathrm{k}$ ' is propagation constants in fee space and wall respectively

- $\theta_{t}, \theta_{i}, 1, \mathrm{~b}$ is defined in [16].

The E-field that is transmitted through the wall will be attenuated and phaseshifted according to (4): 


$$
\begin{gathered}
E(z)=E(0)+e^{-\alpha z} e^{-j \beta z} \\
\alpha \cong \frac{\sigma}{2} \sqrt{\frac{\mu}{\varepsilon}} \quad \beta \cong \omega \sqrt{\mu \varepsilon}
\end{gathered}
$$

Where:

- $E(z)$ is the E-field component in distance $\mathrm{z}$ from the source $E(0)$

- Material permeability $(\mu)$

- Material permittivity $(\varepsilon)$

- Material conductivity $(\sigma)$

Some examples of common used material-parameters are given in Table 1 together with some examples of wall-attenuations for a ray (signal) penetrating a $15 \mathrm{~cm}$ thick wall in an angle of 30 degrees [4].

\subsection{The Model}

A passive repeater model [17] can be constructed by combining the equations discussed in the previous Sections (1)-(4) and using these to describe the superimposed signal behind a wall as illustrated in Figure 4. To simplify the model it uses two rays connecting the Google 'Loon' with a LTE mobile phone. One ray models the direct signal passing through the wall which means it is attenuated and phase-shifted by the complex impedance of the wall. The other ray hits the outdoor antenna of the passive repeater and is retransmitted inside the home. These rays are then combined into one signal which is received by a LTE mobile phone.

Some assumptions have been used to construct this model, these are: the walls are homogenous plates, no reflections from the home walls take place, the multi-reflections inside the wall are negligible, and the walls electric parameters can be considered constant in the signal bandwidth. Most of these assumptions are fulfilled by the house-types used in rural Africa. In addition, it is noted that the passive repeater system is "reciprocal" which means that

Table 1 Estimated wall losses (in $\mathrm{dB}$ ) as a function of electrical parameters for building materials [4]

\begin{tabular}{lccc}
\hline Material & Permittivity & Conductivity $[\mathrm{S} / \mathrm{m}]$ & Wall Loss [dB] \\
\hline Semi dry clay & 10 & 0.02 & 6.3 \\
Semi dry sand & 10 & 0.01 & 5.8 \\
Brick wall & 4.5 & 0.02 & 4.2 \\
Reinforced Concrete & 7 & 0.1 & 12.0 \\
Wood (wet) & 6 & 0.01 & 3.9 \\
\hline
\end{tabular}




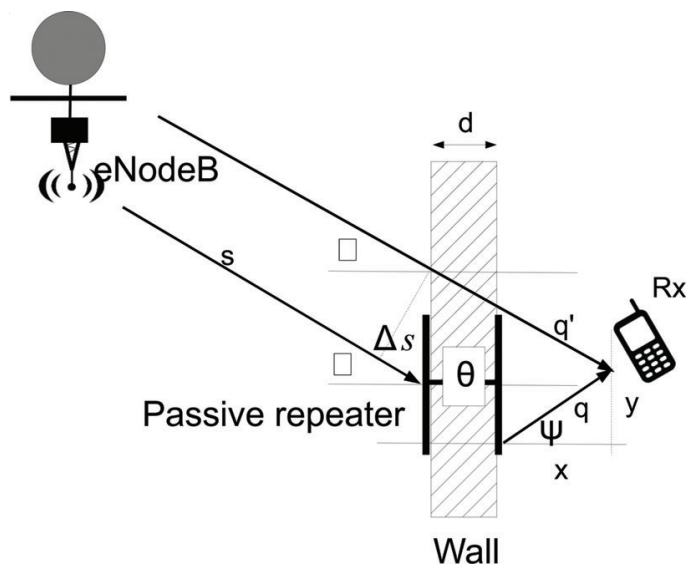

Figure 4 The physical context for the passive repeater simulation model.

the model covers two way communications, i.e. from the eNodeB to the LTE mobile phone and the other way as well.

The ray from the eNodeB to the LTE mobile phone can be modeled by using Equation (3) and Equation (5) describing the far-field from a dipole antenna. Combining these equations yields Equation (6).

$$
\begin{gathered}
\bar{E} \cong \frac{\sqrt{30 P_{t} G_{t}}}{r} e^{-j \beta r} \\
\bar{E}_{r d}=\frac{\sqrt{30 P_{t} G_{t}}}{s+q^{\prime}}|T| e^{-j\left(\beta\left(s+q^{\prime}\right)-\theta_{T}\right)}
\end{gathered}
$$

Where $\beta$ is the free-space phase constant, $r$ is the distance, $P_{t}$ is the transmitted power, and $G_{t}$ is the transmitter antenna gain.

By using Friis formula (1) together with (5) Equation (7) can be derived.

$$
\bar{E}_{r r}=\sqrt{\frac{30 P_{t} G_{t}}{L_{c}}} \frac{\lambda}{4 \pi(s+\Delta s) q} \sqrt{G_{r r} G_{r t}} F_{r r}(\phi) F_{r t}(\phi) e^{-j \beta\left(s+\Delta s+q+\sqrt{\varepsilon_{r}} d\right)}
$$

Where $L_{c}$ is phase shifter and cable losses. $G_{r}$ and $G_{t}$ are the respective antenna gains. $\lambda$ is the wavelength. $G_{r r}$ and $G_{r t}$ are gains for the passive repeater antennas respectively. $F_{r r}(\phi)$ and $F_{r t}(\phi)$ are the directionality functions for the passive repeater antennas.

Finally, the signal inside the home can be found by adding the two rays, i.e. Equation (6) and Equation (7). It is noted that the ray passing through 
the passive repeater is multiplied with a phase change term which models the phase-shifter device found in a passive repeater system.

$$
\bar{E}_{r}=\bar{E}_{r d}+\bar{E}_{r r} e^{j \theta}
$$

\section{The Simulated Results}

By implementing Equations (1) to (8) in a mathematical tool a simulation model has been derived. This model has been run with the following parameters:

- Wall thickness is $10 \mathrm{~cm}$.

- eNodeB antenna gain is set to $12 \mathrm{dBi}$.

- Frequency is $2.6 \mathrm{GHz}$. (used for LTE in part of Africa).

- Distance from transmitter to the home is $20 \mathrm{~km}$.

- Passive repeater antenna gains are $20 \mathrm{dBi}$ for the outer antenna and 10 $\mathrm{dBi}$ for indoor antenna.

- Attenuation phase shifter is set to $1.0 \mathrm{~dB}$.

- Angle between the transmitted ray and the house wall normal vector $(\phi)$ is set to 75 degrees.

The first run (Figure 5) was on a "semi-dry clay" wall.

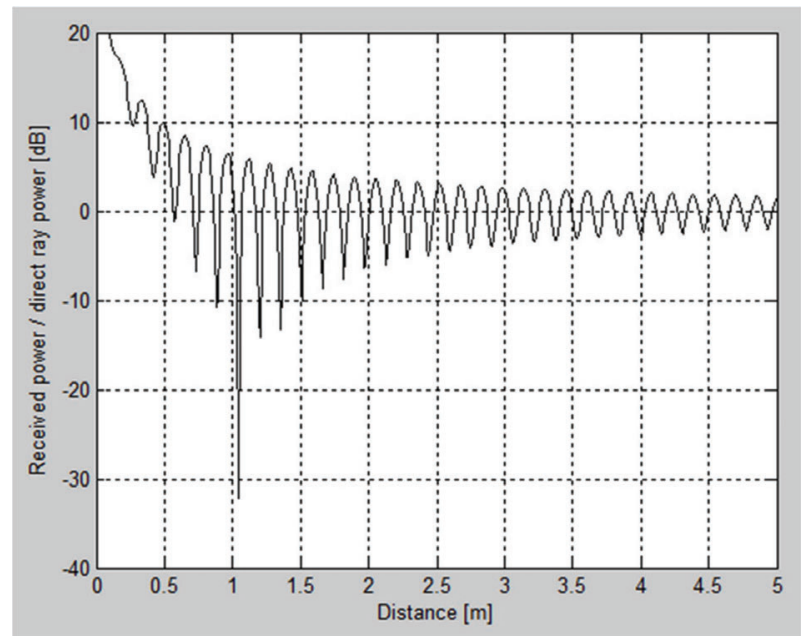

Figure 5 The simulated result for the received power relative to the received power in the direct ray as a function of distance from the wall inside the home. 


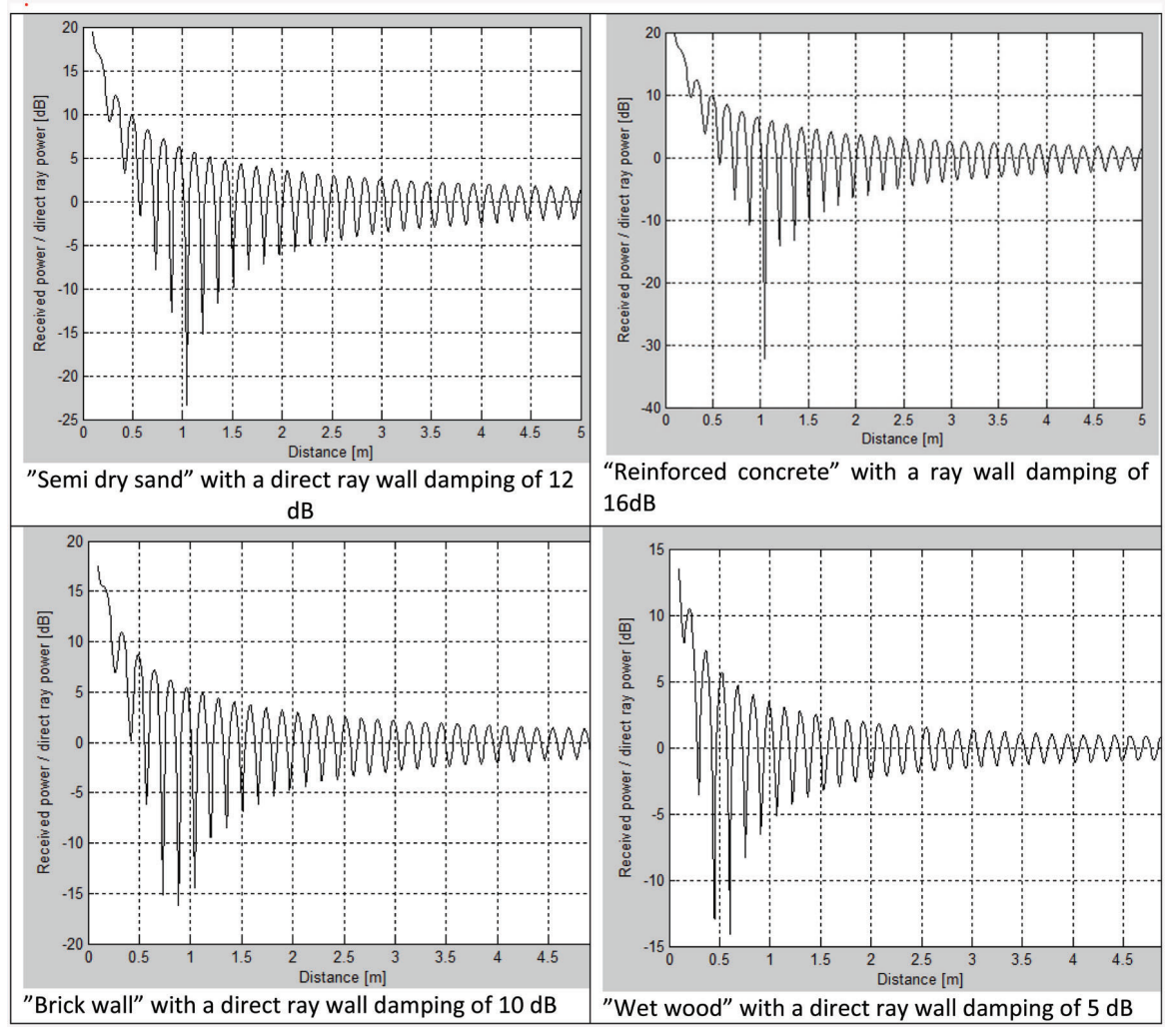

Figure 6 Same as previous figure, but with different choices of building materials.

It is noted that the gain of the outdoor antenna of the passive repeater is set to $20 \mathrm{dBi}$. By using Equation (2) this means that it covers a circular sky area with a diameter of approx. $15 \mathrm{~km}$ which is assumed to be sufficient to cover the drift of the balloons.

The output of the simulation (Figure 5) is the ratio of received power (sum of the rays) divided by the power in the direct ray. It is observed that the power fluctuates as expected because the two rays combines in both constructive and destructive ways. Hence, the signal close to the wall (less than 0.5 meter) is amplified with approximately $10 \mathrm{db}$, whereas this amplification is down to approximately $8 \mathrm{db}$ at a distance of one meter. This gain is the key to overcome the wall damping on the direct ray which is approximately $12 \mathrm{db}$ with the given parameters. Hence a net gain loss of approximately $2 \mathrm{db}$ can be observed. By focusing on the received power at the antenna of the eNodeB from a typical 
LTE mobile phone (Figure 3 ) it is noted that a gain loss of $2 \mathrm{db}$ is acceptable because the signal will still be above $-91 \mathrm{dBm}$. However, without the passive repeater a gain loss of $12 \mathrm{db}$ would move the receive power at the eNodeB below the threshold limit of $-91 \mathrm{dBm}$ which means that transmission would not be possible, i.e. a connection cannot be established.

Plot of output for the same scenario with the wall material set to "semi dry sand", "reinforced concrete", "brick wall", and "wet wood" are shown in Figure 6. It is noted that walls of "reinforced concrete" will have a net-loss of $6 \mathrm{~dB}$ which means that the signal from the LTE mobile phone would not be amplified enough to overcome the $-91 \mathrm{dBm}$ limit (Figure 3). Similarly focusing on walls of "wet wood" the net-loss will be negative, i.e. there exists a net-gain of approximately $2-3 \mathrm{~dB}$. This gain can be used to either achieve a more reliable signal in case of the path degrading factors discussed earlier or it can be used for reducing the complexity and size of the outdoor antenna by lowering its gain with $2-3 \mathrm{~dB}$.

\section{Conclusion}

In general the Google 'Loon' system is able to connect an outdoor LTE mobile phone to its eNodeB base-station. However, as seen from the simulations this connection is at the limits of what is possible for achieving a high LTE datarate communication in most cases. This means that it is not possible for a LTE mobile phone to connect to the eNodeB base-station from inside the homes because the wall-losses bring the signal level below the threshold for communication. Nevertheless, by using a passive repeater it has been shown that the indoor signal level can be raised with approximately the same amount as the wall-loss. This means that the indoor conditions are very similar to the outdoor conditions for wall building materials such as "semi-dry clay", "wet wood", and "brick walls". However, it will not be possible for materials like "reinforced concrete".

From a summary perspective, it has been found that indoor connection is possible by using a passive repeater for most building materials used in rural areas in Africa.

Nonetheless, this solution also has disadvantages such as: the user needs to find the maximum indoor signal, i.e. avoiding the blind spots; the materials for building the passive repeater costs a few hundred dollars, but it is a onetime investment; and the signal is still close to the limit of the of what is necessary to establish a high data-rate LTE connection, i.e. no margin exists. 
In addition, it is noted that this solution offers benefits as it is affordable in the long run, it is sustainable, and it is power neutral, why it fits very well to rural areas in Africa as well as in other rural areas throughout the world.

\section{References}

[1] GSMA Intelligence (2016). The Mobile Economy, Africa. London: GSMA.

[2] Dailymail (2017). Available at: http://www.dailymail.co.uk/science tech/article-2342127/Now-Google-launches-BALLOONS-bid-bring-int ernet-remotest-places-Earth.html

[3] Google Loon (2015) Slideshare. Available at: https://www.slideshare.net/ RichaSingh59/loon-33360993

[4] Lynggaard, P. (2015). Improving Internet Coverage in Rural Africa by Using Passive Repeaters in the Home.

[5] BlueTown (2015) Available at: http://bluetown.com/company/

[6] Simba, F., Mwinyiwiwa, B. M., Mjema, E. M., Trojer, L., and Mvungi, N. H. (2011). Broadband access technologies for rural connectivity in developing countries. Int. J. Res. Rev. Comput. Sci. 2, 312-319.

[7] Gilat Satellite Networks (2015). Extending Mobile Networks Into Rural Areas via Satellite. Petah Tikva: Gilat Satellite Networks

[8] Roslee, M., and Chimheno, R. (2013). Wireless regional access networks: a wise choice for internet connectivity to rural areas of Zimbabwe. Mod. Appl. Sci. 7, 29-31.

[9] Company X (2017). Available at: https://x.company/loon/journey/

[10] CNBC (2017). Available at: https://www.cnbc.com/2017/02/16/googlex-boasts-big-improvement-in-project-loon.html

[11] ETSI (2016). "TS 136101 - V13.3.0 - LTE; Evolved Universal Terrestrial Radio Access (E-UTRA); User Equipment (UE) radio transmission and reception (3GPP TS 36.101 version 13.3.0 Release 13)," ETSI, TS 136 101 - V13.3.0 - LTE, 2016. Sophia Antipolis: ETSI.

[12] Sklar, B. (2002). Digital Communications, Fundamentals and Applications, 2nd Edn. London: Pearson.

[13] Shaw, J. (2013). Radiometry and the Friis transmission equation. Am. J. Phys. 81, 33-37.

[14] NAVAIR Electronic Warfare/Combat System (2012). Electronic Warfare And Radar Systems Engineering Handbook: Technical Information Division. New Delhi: NAVAIR. 
[15] Zaytsev, A. (2013). RF design for the mobiledevices explosion. Cisco Connect. 2013, 36.

[16] Burnside, W. D. (1983). High frequency scattering by a thin lossless dielectric slab. IEEE Trans. Antennas Propagation 31, 104-110.

[17] Hristov, H., Feick, R., and Grote, W. (2001). Improving indoor signal coverage by use of through-wall passive repeaters. IEEE Antennas Propagation 2, 158-161.

[18] ITU. (2011) Available at: https://www.itu.int/ITU-D/ict/facts/2011/ material/ICTFactsFigures2011.pdf

\section{Biography}

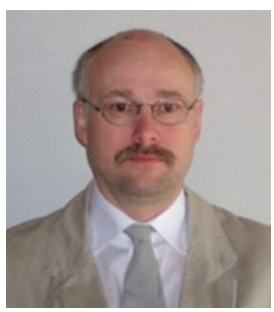

Per Lynggaard is an associate professor in the communication, media, and information systems section at Aalborg University Copenhagen, Denmark. Dr. Lynggaard received his B.Sc. in Electrical Engineering from Technical University of Denmark and his M.Sc. and Ph.D. from Aalborg University.

Dr. Lynggaard has many years' experience of industrial development within a broad range of areas, e.g., signal processing, digital electronics, software development, artificial intelligence, machine learning, SDR and IoT. Through the years, he has given conference presentations, given lectures, and published papers in these and other topics at Technical University of Denmark and at Aalborg University, Denmark. 
